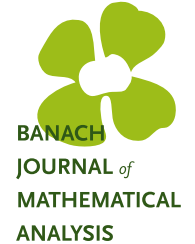

Banach J. Math. Anal. 10 (2016), no. 4, 848-863

http://dx.doi.org/10.1215/17358787-3649722

ISSN: $1735-8787$ (electronic)

http://projecteuclid.org/bjma

\title{
WANDERING SUBSPACES AND FUSION FRAME GENERATORS FOR UNITARY SYSTEMS
}

\author{
AIFANG LIU and PENGTONG LI*
}

Communicated by Z. Páles

\begin{abstract}
This work is inspired by the study of wandering vectors and frame vectors for unitary systems. We investigate the structure and properties of complete wandering subspaces for unitary systems, and, in particular, we consider the unitary systems with a structure similar to wavelet systems. Given a unitary system with a complete wandering subspace, a necessary and sufficient condition for a closed subspace to be a Parseval fusion frame generator is obtained. Moreover, we study the dilation property for Parseval fusion frame generators for unitary groups.
\end{abstract}

\section{InTRODUCTION}

In operator theory, wandering vectors and wandering subspaces have been studied for unitary systems and isometry systems (see [8], [14], [21]). Wavelet theory entails the study of wandering vectors for unitary systems. Dai and Larson [8] showed that orthogonal wavelets can be viewed as wandering vectors for dilationtranslation unitary systems. The connection between multiresolution analysis and the concept of wandering subspaces of unitary operators in Hilbert spaces was given by Goodman, Lee, and Tang [13]. We know that multiresolution analysis plays an important role in wavelet theory. Indeed, the classical construction of wavelets arises from multiresolution analysis. With the development of wavelets, many different aspects of the wavelet theory have been studied. They are useful

Copyright 2016 by the Tusi Mathematical Research Group.

Received Oct. 20, 2015; Accepted Jan. 31, 2016.

${ }^{*}$ Corresponding author.

2010 Mathematics Subject Classification. Primary 42C15; Secondary 42C40, 47D03.

Keywords. unitary system, local commutant, wandering subspace, fusion frame generator, dilation. 


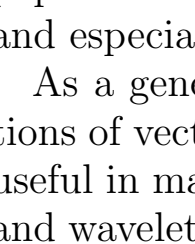

Banach J. Math. Anal. 10 (2016), no. 4, 848-863

http://dx.doi.org/10.1215/17358787-3649722

ISSN: $1735-8787$ (electronic)

http://projecteuclid.org/bjma

\title{
WANDERING SUBSPACES AND FUSION FRAME GENERATORS FOR UNITARY SYSTEMS
}

\author{
AIFANG LIU and PENGTONG LI*
}

Communicated by Z. Páles

\begin{abstract}
This work is inspired by the study of wandering vectors and frame vectors for unitary systems. We investigate the structure and properties of complete wandering subspaces for unitary systems, and, in particular, we consider the unitary systems with a structure similar to wavelet systems. Given a unitary system with a complete wandering subspace, a necessary and sufficient condition for a closed subspace to be a Parseval fusion frame generator is obtained. Moreover, we study the dilation property for Parseval fusion frame generators for unitary groups.
\end{abstract}

\section{InTRODUCTION}

In operator theory, wandering vectors and wandering subspaces have been studied for unitary systems and isometry systems (see [8], [14], [21]). Wavelet theory entails the study of wandering vectors for unitary systems. Dai and Larson [8] showed that orthogonal wavelets can be viewed as wandering vectors for dilationtranslation unitary systems. The connection between multiresolution analysis and the concept of wandering subspaces of unitary operators in Hilbert spaces was given by Goodman, Lee, and Tang [13]. We know that multiresolution analysis plays an important role in wavelet theory. Indeed, the classical construction of wavelets arises from multiresolution analysis. With the development of wavelets, many different aspects of the wavelet theory have been studied. They are useful

Copyright 2016 by the Tusi Mathematical Research Group.

Received Oct. 20, 2015; Accepted Jan. 31, 2016.

${ }^{*}$ Corresponding author.

2010 Mathematics Subject Classification. Primary 42C15; Secondary 42C40, 47D03.

Keywords. unitary system, local commutant, wandering subspace, fusion frame generator, dilation. 


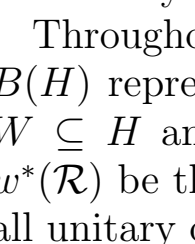

Banach J. Math. Anal. 10 (2016), no. 4, 848-863

http://dx.doi.org/10.1215/17358787-3649722

ISSN: $1735-8787$ (electronic)

http://projecteuclid.org/bjma

\title{
WANDERING SUBSPACES AND FUSION FRAME GENERATORS FOR UNITARY SYSTEMS
}

\author{
AIFANG LIU and PENGTONG LI*
}

Communicated by Z. Páles

\begin{abstract}
This work is inspired by the study of wandering vectors and frame vectors for unitary systems. We investigate the structure and properties of complete wandering subspaces for unitary systems, and, in particular, we consider the unitary systems with a structure similar to wavelet systems. Given a unitary system with a complete wandering subspace, a necessary and sufficient condition for a closed subspace to be a Parseval fusion frame generator is obtained. Moreover, we study the dilation property for Parseval fusion frame generators for unitary groups.
\end{abstract}

\section{InTRODUCTION}

In operator theory, wandering vectors and wandering subspaces have been studied for unitary systems and isometry systems (see [8], [14], [21]). Wavelet theory entails the study of wandering vectors for unitary systems. Dai and Larson [8] showed that orthogonal wavelets can be viewed as wandering vectors for dilationtranslation unitary systems. The connection between multiresolution analysis and the concept of wandering subspaces of unitary operators in Hilbert spaces was given by Goodman, Lee, and Tang [13]. We know that multiresolution analysis plays an important role in wavelet theory. Indeed, the classical construction of wavelets arises from multiresolution analysis. With the development of wavelets, many different aspects of the wavelet theory have been studied. They are useful

Copyright 2016 by the Tusi Mathematical Research Group.

Received Oct. 20, 2015; Accepted Jan. 31, 2016.

${ }^{*}$ Corresponding author.

2010 Mathematics Subject Classification. Primary 42C15; Secondary 42C40, 47D03.

Keywords. unitary system, local commutant, wandering subspace, fusion frame generator, dilation. 


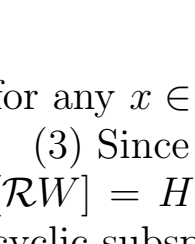

Banach J. Math. Anal. 10 (2016), no. 4, 848-863

http://dx.doi.org/10.1215/17358787-3649722

ISSN: $1735-8787$ (electronic)

http://projecteuclid.org/bjma

\title{
WANDERING SUBSPACES AND FUSION FRAME GENERATORS FOR UNITARY SYSTEMS
}

\author{
AIFANG LIU and PENGTONG LI*
}

Communicated by Z. Páles

\begin{abstract}
This work is inspired by the study of wandering vectors and frame vectors for unitary systems. We investigate the structure and properties of complete wandering subspaces for unitary systems, and, in particular, we consider the unitary systems with a structure similar to wavelet systems. Given a unitary system with a complete wandering subspace, a necessary and sufficient condition for a closed subspace to be a Parseval fusion frame generator is obtained. Moreover, we study the dilation property for Parseval fusion frame generators for unitary groups.
\end{abstract}

\section{InTRODUCTION}

In operator theory, wandering vectors and wandering subspaces have been studied for unitary systems and isometry systems (see [8], [14], [21]). Wavelet theory entails the study of wandering vectors for unitary systems. Dai and Larson [8] showed that orthogonal wavelets can be viewed as wandering vectors for dilationtranslation unitary systems. The connection between multiresolution analysis and the concept of wandering subspaces of unitary operators in Hilbert spaces was given by Goodman, Lee, and Tang [13]. We know that multiresolution analysis plays an important role in wavelet theory. Indeed, the classical construction of wavelets arises from multiresolution analysis. With the development of wavelets, many different aspects of the wavelet theory have been studied. They are useful

Copyright 2016 by the Tusi Mathematical Research Group.

Received Oct. 20, 2015; Accepted Jan. 31, 2016.

${ }^{*}$ Corresponding author.

2010 Mathematics Subject Classification. Primary 42C15; Secondary 42C40, 47D03.

Keywords. unitary system, local commutant, wandering subspace, fusion frame generator, dilation. 


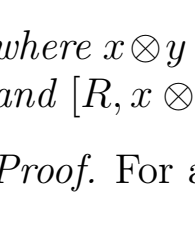

Banach J. Math. Anal. 10 (2016), no. 4, 848-863

http://dx.doi.org/10.1215/17358787-3649722

ISSN: $1735-8787$ (electronic)

http://projecteuclid.org/bjma

\title{
WANDERING SUBSPACES AND FUSION FRAME GENERATORS FOR UNITARY SYSTEMS
}

\author{
AIFANG LIU and PENGTONG LI*
}

Communicated by Z. Páles

\begin{abstract}
This work is inspired by the study of wandering vectors and frame vectors for unitary systems. We investigate the structure and properties of complete wandering subspaces for unitary systems, and, in particular, we consider the unitary systems with a structure similar to wavelet systems. Given a unitary system with a complete wandering subspace, a necessary and sufficient condition for a closed subspace to be a Parseval fusion frame generator is obtained. Moreover, we study the dilation property for Parseval fusion frame generators for unitary groups.
\end{abstract}

\section{InTRODUCTION}

In operator theory, wandering vectors and wandering subspaces have been studied for unitary systems and isometry systems (see [8], [14], [21]). Wavelet theory entails the study of wandering vectors for unitary systems. Dai and Larson [8] showed that orthogonal wavelets can be viewed as wandering vectors for dilationtranslation unitary systems. The connection between multiresolution analysis and the concept of wandering subspaces of unitary operators in Hilbert spaces was given by Goodman, Lee, and Tang [13]. We know that multiresolution analysis plays an important role in wavelet theory. Indeed, the classical construction of wavelets arises from multiresolution analysis. With the development of wavelets, many different aspects of the wavelet theory have been studied. They are useful

Copyright 2016 by the Tusi Mathematical Research Group.

Received Oct. 20, 2015; Accepted Jan. 31, 2016.

${ }^{*}$ Corresponding author.

2010 Mathematics Subject Classification. Primary 42C15; Secondary 42C40, 47D03.

Keywords. unitary system, local commutant, wandering subspace, fusion frame generator, dilation. 


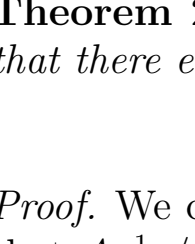

Banach J. Math. Anal. 10 (2016), no. 4, 848-863

http://dx.doi.org/10.1215/17358787-3649722

ISSN: $1735-8787$ (electronic)

http://projecteuclid.org/bjma

\title{
WANDERING SUBSPACES AND FUSION FRAME GENERATORS FOR UNITARY SYSTEMS
}

\author{
AIFANG LIU and PENGTONG LI*
}

Communicated by Z. Páles

\begin{abstract}
This work is inspired by the study of wandering vectors and frame vectors for unitary systems. We investigate the structure and properties of complete wandering subspaces for unitary systems, and, in particular, we consider the unitary systems with a structure similar to wavelet systems. Given a unitary system with a complete wandering subspace, a necessary and sufficient condition for a closed subspace to be a Parseval fusion frame generator is obtained. Moreover, we study the dilation property for Parseval fusion frame generators for unitary groups.
\end{abstract}

\section{InTRODUCTION}

In operator theory, wandering vectors and wandering subspaces have been studied for unitary systems and isometry systems (see [8], [14], [21]). Wavelet theory entails the study of wandering vectors for unitary systems. Dai and Larson [8] showed that orthogonal wavelets can be viewed as wandering vectors for dilationtranslation unitary systems. The connection between multiresolution analysis and the concept of wandering subspaces of unitary operators in Hilbert spaces was given by Goodman, Lee, and Tang [13]. We know that multiresolution analysis plays an important role in wavelet theory. Indeed, the classical construction of wavelets arises from multiresolution analysis. With the development of wavelets, many different aspects of the wavelet theory have been studied. They are useful

Copyright 2016 by the Tusi Mathematical Research Group.

Received Oct. 20, 2015; Accepted Jan. 31, 2016.

${ }^{*}$ Corresponding author.

2010 Mathematics Subject Classification. Primary 42C15; Secondary 42C40, 47D03.

Keywords. unitary system, local commutant, wandering subspace, fusion frame generator, dilation. 


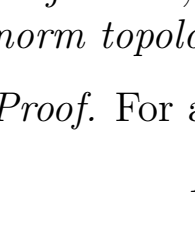

Banach J. Math. Anal. 10 (2016), no. 4, 848-863

http://dx.doi.org/10.1215/17358787-3649722

ISSN: $1735-8787$ (electronic)

http://projecteuclid.org/bjma

\title{
WANDERING SUBSPACES AND FUSION FRAME GENERATORS FOR UNITARY SYSTEMS
}

\author{
AIFANG LIU and PENGTONG LI*
}

Communicated by Z. Páles

\begin{abstract}
This work is inspired by the study of wandering vectors and frame vectors for unitary systems. We investigate the structure and properties of complete wandering subspaces for unitary systems, and, in particular, we consider the unitary systems with a structure similar to wavelet systems. Given a unitary system with a complete wandering subspace, a necessary and sufficient condition for a closed subspace to be a Parseval fusion frame generator is obtained. Moreover, we study the dilation property for Parseval fusion frame generators for unitary groups.
\end{abstract}

\section{InTRODUCTION}

In operator theory, wandering vectors and wandering subspaces have been studied for unitary systems and isometry systems (see [8], [14], [21]). Wavelet theory entails the study of wandering vectors for unitary systems. Dai and Larson [8] showed that orthogonal wavelets can be viewed as wandering vectors for dilationtranslation unitary systems. The connection between multiresolution analysis and the concept of wandering subspaces of unitary operators in Hilbert spaces was given by Goodman, Lee, and Tang [13]. We know that multiresolution analysis plays an important role in wavelet theory. Indeed, the classical construction of wavelets arises from multiresolution analysis. With the development of wavelets, many different aspects of the wavelet theory have been studied. They are useful

Copyright 2016 by the Tusi Mathematical Research Group.

Received Oct. 20, 2015; Accepted Jan. 31, 2016.

${ }^{*}$ Corresponding author.

2010 Mathematics Subject Classification. Primary 42C15; Secondary 42C40, 47D03.

Keywords. unitary system, local commutant, wandering subspace, fusion frame generator, dilation. 


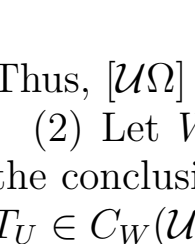

Banach J. Math. Anal. 10 (2016), no. 4, 848-863

http://dx.doi.org/10.1215/17358787-3649722

ISSN: $1735-8787$ (electronic)

http://projecteuclid.org/bjma

\title{
WANDERING SUBSPACES AND FUSION FRAME GENERATORS FOR UNITARY SYSTEMS
}

\author{
AIFANG LIU and PENGTONG LI*
}

Communicated by Z. Páles

\begin{abstract}
This work is inspired by the study of wandering vectors and frame vectors for unitary systems. We investigate the structure and properties of complete wandering subspaces for unitary systems, and, in particular, we consider the unitary systems with a structure similar to wavelet systems. Given a unitary system with a complete wandering subspace, a necessary and sufficient condition for a closed subspace to be a Parseval fusion frame generator is obtained. Moreover, we study the dilation property for Parseval fusion frame generators for unitary groups.
\end{abstract}

\section{InTRODUCTION}

In operator theory, wandering vectors and wandering subspaces have been studied for unitary systems and isometry systems (see [8], [14], [21]). Wavelet theory entails the study of wandering vectors for unitary systems. Dai and Larson [8] showed that orthogonal wavelets can be viewed as wandering vectors for dilationtranslation unitary systems. The connection between multiresolution analysis and the concept of wandering subspaces of unitary operators in Hilbert spaces was given by Goodman, Lee, and Tang [13]. We know that multiresolution analysis plays an important role in wavelet theory. Indeed, the classical construction of wavelets arises from multiresolution analysis. With the development of wavelets, many different aspects of the wavelet theory have been studied. They are useful

Copyright 2016 by the Tusi Mathematical Research Group.

Received Oct. 20, 2015; Accepted Jan. 31, 2016.

${ }^{*}$ Corresponding author.

2010 Mathematics Subject Classification. Primary 42C15; Secondary 42C40, 47D03.

Keywords. unitary system, local commutant, wandering subspace, fusion frame generator, dilation. 


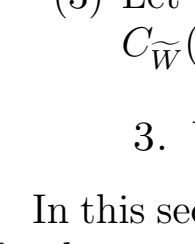

Banach J. Math. Anal. 10 (2016), no. 4, 848-863

http://dx.doi.org/10.1215/17358787-3649722

ISSN: $1735-8787$ (electronic)

http://projecteuclid.org/bjma

\title{
WANDERING SUBSPACES AND FUSION FRAME GENERATORS FOR UNITARY SYSTEMS
}

\author{
AIFANG LIU and PENGTONG LI*
}

Communicated by Z. Páles

\begin{abstract}
This work is inspired by the study of wandering vectors and frame vectors for unitary systems. We investigate the structure and properties of complete wandering subspaces for unitary systems, and, in particular, we consider the unitary systems with a structure similar to wavelet systems. Given a unitary system with a complete wandering subspace, a necessary and sufficient condition for a closed subspace to be a Parseval fusion frame generator is obtained. Moreover, we study the dilation property for Parseval fusion frame generators for unitary groups.
\end{abstract}

\section{InTRODUCTION}

In operator theory, wandering vectors and wandering subspaces have been studied for unitary systems and isometry systems (see [8], [14], [21]). Wavelet theory entails the study of wandering vectors for unitary systems. Dai and Larson [8] showed that orthogonal wavelets can be viewed as wandering vectors for dilationtranslation unitary systems. The connection between multiresolution analysis and the concept of wandering subspaces of unitary operators in Hilbert spaces was given by Goodman, Lee, and Tang [13]. We know that multiresolution analysis plays an important role in wavelet theory. Indeed, the classical construction of wavelets arises from multiresolution analysis. With the development of wavelets, many different aspects of the wavelet theory have been studied. They are useful

Copyright 2016 by the Tusi Mathematical Research Group.

Received Oct. 20, 2015; Accepted Jan. 31, 2016.

${ }^{*}$ Corresponding author.

2010 Mathematics Subject Classification. Primary 42C15; Secondary 42C40, 47D03.

Keywords. unitary system, local commutant, wandering subspace, fusion frame generator, dilation. 


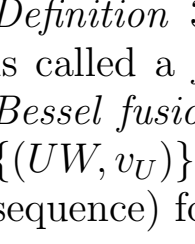

Banach J. Math. Anal. 10 (2016), no. 4, 848-863

http://dx.doi.org/10.1215/17358787-3649722

ISSN: $1735-8787$ (electronic)

http://projecteuclid.org/bjma

\title{
WANDERING SUBSPACES AND FUSION FRAME GENERATORS FOR UNITARY SYSTEMS
}

\author{
AIFANG LIU and PENGTONG LI*
}

Communicated by Z. Páles

\begin{abstract}
This work is inspired by the study of wandering vectors and frame vectors for unitary systems. We investigate the structure and properties of complete wandering subspaces for unitary systems, and, in particular, we consider the unitary systems with a structure similar to wavelet systems. Given a unitary system with a complete wandering subspace, a necessary and sufficient condition for a closed subspace to be a Parseval fusion frame generator is obtained. Moreover, we study the dilation property for Parseval fusion frame generators for unitary groups.
\end{abstract}

\section{InTRODUCTION}

In operator theory, wandering vectors and wandering subspaces have been studied for unitary systems and isometry systems (see [8], [14], [21]). Wavelet theory entails the study of wandering vectors for unitary systems. Dai and Larson [8] showed that orthogonal wavelets can be viewed as wandering vectors for dilationtranslation unitary systems. The connection between multiresolution analysis and the concept of wandering subspaces of unitary operators in Hilbert spaces was given by Goodman, Lee, and Tang [13]. We know that multiresolution analysis plays an important role in wavelet theory. Indeed, the classical construction of wavelets arises from multiresolution analysis. With the development of wavelets, many different aspects of the wavelet theory have been studied. They are useful

Copyright 2016 by the Tusi Mathematical Research Group.

Received Oct. 20, 2015; Accepted Jan. 31, 2016.

${ }^{*}$ Corresponding author.

2010 Mathematics Subject Classification. Primary 42C15; Secondary 42C40, 47D03.

Keywords. unitary system, local commutant, wandering subspace, fusion frame generator, dilation. 


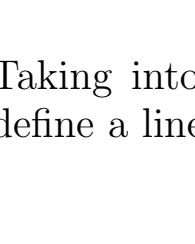

Banach J. Math. Anal. 10 (2016), no. 4, 848-863

http://dx.doi.org/10.1215/17358787-3649722

ISSN: $1735-8787$ (electronic)

http://projecteuclid.org/bjma

\title{
WANDERING SUBSPACES AND FUSION FRAME GENERATORS FOR UNITARY SYSTEMS
}

\author{
AIFANG LIU and PENGTONG LI*
}

Communicated by Z. Páles

\begin{abstract}
This work is inspired by the study of wandering vectors and frame vectors for unitary systems. We investigate the structure and properties of complete wandering subspaces for unitary systems, and, in particular, we consider the unitary systems with a structure similar to wavelet systems. Given a unitary system with a complete wandering subspace, a necessary and sufficient condition for a closed subspace to be a Parseval fusion frame generator is obtained. Moreover, we study the dilation property for Parseval fusion frame generators for unitary groups.
\end{abstract}

\section{InTRODUCTION}

In operator theory, wandering vectors and wandering subspaces have been studied for unitary systems and isometry systems (see [8], [14], [21]). Wavelet theory entails the study of wandering vectors for unitary systems. Dai and Larson [8] showed that orthogonal wavelets can be viewed as wandering vectors for dilationtranslation unitary systems. The connection between multiresolution analysis and the concept of wandering subspaces of unitary operators in Hilbert spaces was given by Goodman, Lee, and Tang [13]. We know that multiresolution analysis plays an important role in wavelet theory. Indeed, the classical construction of wavelets arises from multiresolution analysis. With the development of wavelets, many different aspects of the wavelet theory have been studied. They are useful

Copyright 2016 by the Tusi Mathematical Research Group.

Received Oct. 20, 2015; Accepted Jan. 31, 2016.

${ }^{*}$ Corresponding author.

2010 Mathematics Subject Classification. Primary 42C15; Secondary 42C40, 47D03.

Keywords. unitary system, local commutant, wandering subspace, fusion frame generator, dilation. 


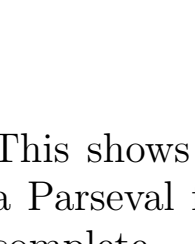

Banach J. Math. Anal. 10 (2016), no. 4, 848-863

http://dx.doi.org/10.1215/17358787-3649722

ISSN: $1735-8787$ (electronic)

http://projecteuclid.org/bjma

\title{
WANDERING SUBSPACES AND FUSION FRAME GENERATORS FOR UNITARY SYSTEMS
}

\author{
AIFANG LIU and PENGTONG LI*
}

Communicated by Z. Páles

\begin{abstract}
This work is inspired by the study of wandering vectors and frame vectors for unitary systems. We investigate the structure and properties of complete wandering subspaces for unitary systems, and, in particular, we consider the unitary systems with a structure similar to wavelet systems. Given a unitary system with a complete wandering subspace, a necessary and sufficient condition for a closed subspace to be a Parseval fusion frame generator is obtained. Moreover, we study the dilation property for Parseval fusion frame generators for unitary groups.
\end{abstract}

\section{InTRODUCTION}

In operator theory, wandering vectors and wandering subspaces have been studied for unitary systems and isometry systems (see [8], [14], [21]). Wavelet theory entails the study of wandering vectors for unitary systems. Dai and Larson [8] showed that orthogonal wavelets can be viewed as wandering vectors for dilationtranslation unitary systems. The connection between multiresolution analysis and the concept of wandering subspaces of unitary operators in Hilbert spaces was given by Goodman, Lee, and Tang [13]. We know that multiresolution analysis plays an important role in wavelet theory. Indeed, the classical construction of wavelets arises from multiresolution analysis. With the development of wavelets, many different aspects of the wavelet theory have been studied. They are useful

Copyright 2016 by the Tusi Mathematical Research Group.

Received Oct. 20, 2015; Accepted Jan. 31, 2016.

${ }^{*}$ Corresponding author.

2010 Mathematics Subject Classification. Primary 42C15; Secondary 42C40, 47D03.

Keywords. unitary system, local commutant, wandering subspace, fusion frame generator, dilation. 


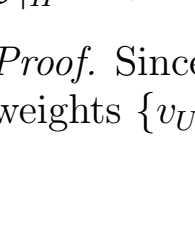

Banach J. Math. Anal. 10 (2016), no. 4, 848-863

http://dx.doi.org/10.1215/17358787-3649722

ISSN: $1735-8787$ (electronic)

http://projecteuclid.org/bjma

\title{
WANDERING SUBSPACES AND FUSION FRAME GENERATORS FOR UNITARY SYSTEMS
}

\author{
AIFANG LIU and PENGTONG LI*
}

Communicated by Z. Páles

\begin{abstract}
This work is inspired by the study of wandering vectors and frame vectors for unitary systems. We investigate the structure and properties of complete wandering subspaces for unitary systems, and, in particular, we consider the unitary systems with a structure similar to wavelet systems. Given a unitary system with a complete wandering subspace, a necessary and sufficient condition for a closed subspace to be a Parseval fusion frame generator is obtained. Moreover, we study the dilation property for Parseval fusion frame generators for unitary groups.
\end{abstract}

\section{InTRODUCTION}

In operator theory, wandering vectors and wandering subspaces have been studied for unitary systems and isometry systems (see [8], [14], [21]). Wavelet theory entails the study of wandering vectors for unitary systems. Dai and Larson [8] showed that orthogonal wavelets can be viewed as wandering vectors for dilationtranslation unitary systems. The connection between multiresolution analysis and the concept of wandering subspaces of unitary operators in Hilbert spaces was given by Goodman, Lee, and Tang [13]. We know that multiresolution analysis plays an important role in wavelet theory. Indeed, the classical construction of wavelets arises from multiresolution analysis. With the development of wavelets, many different aspects of the wavelet theory have been studied. They are useful

Copyright 2016 by the Tusi Mathematical Research Group.

Received Oct. 20, 2015; Accepted Jan. 31, 2016.

${ }^{*}$ Corresponding author.

2010 Mathematics Subject Classification. Primary 42C15; Secondary 42C40, 47D03.

Keywords. unitary system, local commutant, wandering subspace, fusion frame generator, dilation. 


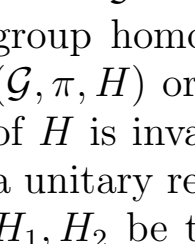

Banach J. Math. Anal. 10 (2016), no. 4, 848-863

http://dx.doi.org/10.1215/17358787-3649722

ISSN: $1735-8787$ (electronic)

http://projecteuclid.org/bjma

\title{
WANDERING SUBSPACES AND FUSION FRAME GENERATORS FOR UNITARY SYSTEMS
}

\author{
AIFANG LIU and PENGTONG LI*
}

Communicated by Z. Páles

\begin{abstract}
This work is inspired by the study of wandering vectors and frame vectors for unitary systems. We investigate the structure and properties of complete wandering subspaces for unitary systems, and, in particular, we consider the unitary systems with a structure similar to wavelet systems. Given a unitary system with a complete wandering subspace, a necessary and sufficient condition for a closed subspace to be a Parseval fusion frame generator is obtained. Moreover, we study the dilation property for Parseval fusion frame generators for unitary groups.
\end{abstract}

\section{InTRODUCTION}

In operator theory, wandering vectors and wandering subspaces have been studied for unitary systems and isometry systems (see [8], [14], [21]). Wavelet theory entails the study of wandering vectors for unitary systems. Dai and Larson [8] showed that orthogonal wavelets can be viewed as wandering vectors for dilationtranslation unitary systems. The connection between multiresolution analysis and the concept of wandering subspaces of unitary operators in Hilbert spaces was given by Goodman, Lee, and Tang [13]. We know that multiresolution analysis plays an important role in wavelet theory. Indeed, the classical construction of wavelets arises from multiresolution analysis. With the development of wavelets, many different aspects of the wavelet theory have been studied. They are useful

Copyright 2016 by the Tusi Mathematical Research Group.

Received Oct. 20, 2015; Accepted Jan. 31, 2016.

${ }^{*}$ Corresponding author.

2010 Mathematics Subject Classification. Primary 42C15; Secondary 42C40, 47D03.

Keywords. unitary system, local commutant, wandering subspace, fusion frame generator, dilation. 


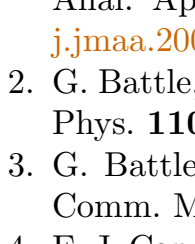

Banach J. Math. Anal. 10 (2016), no. 4, 848-863

http://dx.doi.org/10.1215/17358787-3649722

ISSN: $1735-8787$ (electronic)

http://projecteuclid.org/bjma

\title{
WANDERING SUBSPACES AND FUSION FRAME GENERATORS FOR UNITARY SYSTEMS
}

\author{
AIFANG LIU and PENGTONG LI*
}

Communicated by Z. Páles

\begin{abstract}
This work is inspired by the study of wandering vectors and frame vectors for unitary systems. We investigate the structure and properties of complete wandering subspaces for unitary systems, and, in particular, we consider the unitary systems with a structure similar to wavelet systems. Given a unitary system with a complete wandering subspace, a necessary and sufficient condition for a closed subspace to be a Parseval fusion frame generator is obtained. Moreover, we study the dilation property for Parseval fusion frame generators for unitary groups.
\end{abstract}

\section{InTRODUCTION}

In operator theory, wandering vectors and wandering subspaces have been studied for unitary systems and isometry systems (see [8], [14], [21]). Wavelet theory entails the study of wandering vectors for unitary systems. Dai and Larson [8] showed that orthogonal wavelets can be viewed as wandering vectors for dilationtranslation unitary systems. The connection between multiresolution analysis and the concept of wandering subspaces of unitary operators in Hilbert spaces was given by Goodman, Lee, and Tang [13]. We know that multiresolution analysis plays an important role in wavelet theory. Indeed, the classical construction of wavelets arises from multiresolution analysis. With the development of wavelets, many different aspects of the wavelet theory have been studied. They are useful

Copyright 2016 by the Tusi Mathematical Research Group.

Received Oct. 20, 2015; Accepted Jan. 31, 2016.

${ }^{*}$ Corresponding author.

2010 Mathematics Subject Classification. Primary 42C15; Secondary 42C40, 47D03.

Keywords. unitary system, local commutant, wandering subspace, fusion frame generator, dilation. 


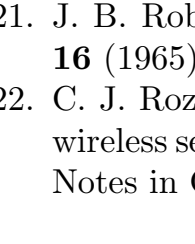

Banach J. Math. Anal. 10 (2016), no. 4, 848-863

http://dx.doi.org/10.1215/17358787-3649722

ISSN: $1735-8787$ (electronic)

http://projecteuclid.org/bjma

\title{
WANDERING SUBSPACES AND FUSION FRAME GENERATORS FOR UNITARY SYSTEMS
}

\author{
AIFANG LIU and PENGTONG LI*
}

Communicated by Z. Páles

\begin{abstract}
This work is inspired by the study of wandering vectors and frame vectors for unitary systems. We investigate the structure and properties of complete wandering subspaces for unitary systems, and, in particular, we consider the unitary systems with a structure similar to wavelet systems. Given a unitary system with a complete wandering subspace, a necessary and sufficient condition for a closed subspace to be a Parseval fusion frame generator is obtained. Moreover, we study the dilation property for Parseval fusion frame generators for unitary groups.
\end{abstract}

\section{InTRODUCTION}

In operator theory, wandering vectors and wandering subspaces have been studied for unitary systems and isometry systems (see [8], [14], [21]). Wavelet theory entails the study of wandering vectors for unitary systems. Dai and Larson [8] showed that orthogonal wavelets can be viewed as wandering vectors for dilationtranslation unitary systems. The connection between multiresolution analysis and the concept of wandering subspaces of unitary operators in Hilbert spaces was given by Goodman, Lee, and Tang [13]. We know that multiresolution analysis plays an important role in wavelet theory. Indeed, the classical construction of wavelets arises from multiresolution analysis. With the development of wavelets, many different aspects of the wavelet theory have been studied. They are useful

Copyright 2016 by the Tusi Mathematical Research Group.

Received Oct. 20, 2015; Accepted Jan. 31, 2016.

${ }^{*}$ Corresponding author.

2010 Mathematics Subject Classification. Primary 42C15; Secondary 42C40, 47D03.

Keywords. unitary system, local commutant, wandering subspace, fusion frame generator, dilation. 